\title{
Avaliação da tireóide em pacientes portadores de psoríase atendidos em um serviço de referência do estado do Pará
}

\author{
Evaluation of the thyroid in patients with psoriasis attended on a reference center on the \\ state of Pará
}
Evaluación de tiroides en pacientes de cuidado de psoriasis asistidos en un servicio de referencia del estado de Pará

Bruna Lima de Oliveira ${ }^{1 *}$, Carlos Victor da Silva Nascimento, Maitê Silva Martins Gadelha, Francisca Regina Oliveira Carneiro'.

\section{RESUMO}

Objetivo: Avaliar as principais disfunções tireoideanas em pacientes portadores de psoríase. Métodos: Tratase de um estudo clínico, transversal e prospectivo. Uma amostra de 40 pacientes com psoríase e 20 controles foram utilizados. O trabalho foi aprovado pelo Comitê de Ética em Pesquisa Resultados: Houve uma diferença significativa $(p<0.0001)$ entre as idades do grupo de pacientes com psoríase quando comparadas ao grupo controle. Os níveis de T4 livre estavam alterados em $38.3 \%$ e foi obtida uma média de $1.6 \pm 2.1$. O grupo controle apresentou uma média de $5.1 \pm 3.7$, com uma diferença significativa $(p<0.0004)$ entre os grupos. Uma diferença considerável foi observada nos níveis de anti-tiroperoxidase (anti-TPO), entre os dois grupos $(p<0.0015)$. Conclusão: Portanto, houve uma relação importante entre os níveis séricos aumentados de anti-TPO em pacientes portadores de psoríase, quando comparados ao grupo controle. Também foi observado um aumento considerável nos níveis de T4 livre entre os pacientes desse grupo. Não foram observadas relação entre o Índice de Severidade da Psoríase por Área (PASI) e disfunções tireoideanas.

Palavras-chave: Dermatologia, Psoríase, Tireóide.

\begin{abstract}
Objective: To observe thyroid dysfunctions in patients with psoriasis. Methods: It is a clinical, transversal and prospective study. A sample of 40 patients with psoriasis and 20 controls were used. The study was approved by the Research Ethics Committee Results: There was a significant difference $(p<0.0001)$ between the ages of patients in the psoriasis group when compared to the control group. Free T4 was altered in $38.3 \%$ and obtained an average of $1.6 \pm 2.1$. The control group presented a mean of $5.1 \pm 3.7$, with a significant difference $(p<0.0004)$ between the groups. A significant difference was observed in the thyroid peroxidase antibody (antiTPO) levels between the groups, $(p<0.0015)$. Conclusion: Therefore, that there is a significant relationship between higher levels of anti-TPO in patients with psoriasis when compared to the control group. There was a significant increase in free T4 levels among patients in this group. No significant relationships were found between Psoriasis Area Severity Index (PASI) and thyroid changes.
\end{abstract}

Key words: Dermatology, Psoriasis, Thyroid.

\section{RESUMEN}

Objetivo: Evaluar la disfunción tiroidea en pacientes con psoriasis. Métodos: Este es un estudio clínico prospectivo, transversal. Se utilizó una muestra de 40 pacientes con psoriasis y 20 controles. El estudio fue aprobado por el Comité de Ética en Investigación. Resultados: Hubo una diferencia significativa $(p \quad i<0,0001)$ entre las edades del grupo de pacientes con psoriasis en comparación con el grupo de control. Los niveles de T4 libre se alteraron en un $38,3 \%$ y se obtuvo un promedio de 1,6 $\pm 2,1$. El grupo control presentó un promedio de $5.1 \pm 3.7$, con una diferencia significativa $(p<0.0004)$ entre los grupos. Se observó una diferencia

${ }^{1}$ Universidade do Estado do Pará (UEPA), Belém - PA. *E-mail: oliveira.bruna@hotmail.com 
considerable en los niveles de anti-tireoperoxidase (anti-TPO) entre los dos grupos $(p<0.0015)$. Conclusión: Se ha observado una relación importante entre el aumento de los niveles séricos de anti-TPO en pacientes con psoriasis en comparación con el grupo control. También se objetivó un aumento considerable en los niveles de T4 libre entre los pacientes de este grupo. No se observó relación entre Índice de la Severidad del Área de Psoriasis (PASI) y disfunción tiroidea.

Palabras clave: Dermatologia, Psoriasis, Tiroides.

\section{INTRODUÇÃO}

Psoríase é uma doença crônica e inflamatória imunomediada por linfócitos $\mathrm{T}$, a qual acomete cerca de $2 \%$ da população mundial, tendo variações de acordo com a etnia e fatores epidemiológicos, sendo mais comum em caucasianos e não tendo diferença significativa entre os sexos (CATHER JC, et al., 2017; HERNÁNDEZVÁSQUEZ A, et al., 2017). Clinicamente a lesão clássica da doença se caracteriza por placas eritematosas cobertas por escamas brancas acinzentadas, mais comumente em locais como cotovelos, joelhos, região lombar e umbilical. Todavia, sabe-se que a psoríase não se trata apenas de uma doença dermatológica, mas sim sistêmica, a qual o maior acometimento descrito se refere as articulações e unhas (SCHÖN MP e BOEHNCKE H, 2005).

A fisiopatologia da doença está ligada à múltiplos fatores, tais como genéticos e ambientais os quais podem dar início à doença a partir de diversos mecanismos de início, como alterações hormonais, estresse emocional, fumo, infeções, variações sazonais e lesões. Além disso, a psoríase é relacionada com múltiplas outras doenças sistêmicas, como síndrome metabólica, dislipidemias, resistência insulínica, diabetes, alterações cardiovasculares e alterações respiratórias incluindo asma. (ROMAN II, et al., 2016).

O papel hormonal na gênese da psoríase é de suma importância, visto que diversas alterações e modulações hormonais foram observadas na fisiopatologia da doença. O sistema hormonal tireoidiano possui uma relação incerta com a gênese da psoríase. Sabe-se que seus hormônios possuem receptores na pele os quais estimulam a proliferação e diferenciação celular, todavia o seu papel no desenvolvimento da doença ainda permanece incerto (ROMAN II, et al., 2016).

Por outro lado, a associação da psoríase com doenças autoimunes já foi constatada. Pacientes já diagnosticados com uma doença autoimune tem uma chance maior de desenvolver uma segunda ou terceira doença ligada à autoimunidade (WU JJ, et al., 2012).

Existem diversas formas de autoimunidade tireoidiana. Notavelmente a mais comum é a tireoidite de Hashimoto, a qual é também é a maior causa de hipotireoidismo, afetando cerca de $2 \%$ na população mundial feminina e $0,2 \%$ masculina (SANTORO D, et al., 2017). Por outro lado, em pacientes com psoríase, existe uma prevalência similar entre os sexos, deixando de ser evidenciada a notória prevalência feminina (VASSILATOU E, et al., 2017). Uma associação entre a tireoidite de Hashimoto e a psoríase vulgar foi observada e tida como significativa, assim como na psoríase artrítica (KIGURADZE T, et al., 2017).

Alterações clínicas e hormonais em pacientes com psoríase já foram observadas quando relacionadas com disfunções tireoidianas, como uma variação dos hormônios tireoidianos em períodos de ativação da psoríase. Além disso, pacientes com psoríase tendem a ter níveis de hormônio estimulante da tireoide (TSH) menores do que pacientes sem a doença (VASSILATOU E, et al., 2017). Por outro lado, pacientes com psoríase tendem a possuir níveis de T4 mais elevados. (GUL U, et al., 2009). Assim como maiores níveis de T3, o qual possui receptores nos queratinóctos e podem estimular o fator de crescimento epidérmico (ROBATI RM, et al., 2013). Além disso, pacientes com maiores níveis de T4 e T3 possuem o score do índice de severidade da psoríase por área (PASI) mais elevado (ARICAN O, et al., 2004).

Observa-se também uma melhor resposta da doença dermatológica quando utilizado medicamentos antitireoidianos como o propiltiouracil em pacientes com psoríase em placa, resultando em melhora clínica e diminuição do PASI. Sendo assim, sugerindo que esta droga modula os linfócitos T presentes nas placas de psoríase (ARICAN O, et al., 2004).

REAS/EJCH | Vol.12(2) | e2378 | DOI: https://doi.org/10.25248/reas.e2378.2020 Página 2 de 8 
Além disso, a droga tem efeito de inibir a ação do hormônio T3 na pele, diminuindo seu efeito proliferativo sob os queratinócitos e assim, evidenciando sua possível relação com a doença. Por fim, observou-se também que a droga é capaz de levar a apoptose das placas psoríaticas (GNANARAJ P, et al., 2015). Ademais, um caso de resolução momentânea da doença pós tireoidectomia com posterior recidiva mais branda da doença e com melhores respostas ao tratamento padrão foi relatado na literatura (KUCHEL J, et al., 2002). Clinicamente, observou-se também que pacientes com alterações tireoidianas tinham períodos de crise dermatológica mais prolongados e com pior resposta aos medicamentos convencionais (LAI YC e YEW YW, 2016).

Em relação à psoríase artrítica, as mulheres com maior número de articulações acometidas, tiveram maior número de casos de disfunção tireoidiana, hipotireoidismo, anti-TPO positivo e alterações ultrassonográficas da glândula (FALLAHI P, et al., 2017). Todavia, a mesma relação ainda não foi identificada nos demais tipos clínicos de psoríase, como na psoríase em placa, necessitando um estudo mais amplo e aprofundado.

Desta forma, diversas alterações hormonais e clínicas relacionadas com alterações tireoidianas já foram descritas na literatura. Todavia, a real relação entre psoríase em placa e alteração da tireoide não foi bem estabelecida cientificamente, necessitando de maior aprofundamento no assunto para um esclarecimento pleno.

\section{MÉTODOS}

Os pacientes da presente pesquisa foram estudados segundo os preceitos da Declaração de Helsinque e do Código de Nuremberg, respeitadas as Normas de Pesquisa Envolvendo Seres Humanos (Res. CNS 466/12) do Conselho Nacional de Saúde. A pesquisa teve início após a submissão e aprovação do projeto pelo Comitê de Ética em Pesquisa da Universidade do Estado do Pará (№ do Parecer: 2.323.446), autorizado pelo diretor responsável pelo Serviço Especializado de Dermatologia da Universidade do Estado do Pará (UEPA), pela orientadora responsável da pesquisa e pelos pacientes, por meio do Termo de Consentimento Livre e Esclarecido (TCLE).

\section{Desenho, local e casuística do estudo}

Trata-se de um estudo clínico, transversal e prospectivo que foi realizado no Serviço Especializado de Dermatologia da Universidade do Estado do Pará (UEPA). A casuística foi de 40 pacientes com psoríase e 20 controles. Esse tamanho amostral foi calculado com base em um Universo de 200 pacientes. Para se chegar a esse tamanho amostral foi utilizada a fórmula para cálculo de amostras com universo inferior a 100.000, dada pela equação: $N=$ d2.p.q.U / e2 (U-1) + d2.p.q. Na qual, adotou-se um universo (U) de y, um percentual de sucesso de 50\%, um percentual de fracasso de $50 \%$, um desvio padrão (d) de 2 e uma margem de erro de $5 \%$.

\section{Critérios de inclusão}

Foram incluídos no estudo pacientes maiores de 18 anos, atendidos no ambulatório de Psoríase do Serviço de Dermatologia da Universidade do Estado do Pará, os quais tiverem diagnóstico confirmado de psoríase por histopatologia prévia ou critérios clínicos e que aceitarem a participação a partir da assinatura do TCLE.

\section{Critérios de Exclusão}

Foram excluídos do estudo paciente menores de 18 ano, os quais não aceitarem os termos de participação da pesquisa e os quais não são cadastrados no Serviço de Dermatologia do Estado do Pará.

\section{Coleta de dados}

A coleta de dados foi realizada por meio de uma entrevista clínica no momento da consulta dermatológica, na qual serão analisados dados epidemiológicos e clínicos como: sexo, idade, escolaridade, ocupação, idade de início e fenótipo clínico da psoríase, duração da doença, tratamentos atuais e anteriores. Posteriormente foi realizado o exame dermatológico com o cálculo do Índice de Área e Severidade da Psoríase (PASI) e aplicado o Dermatological Life Quality Index (DLQI) para avaliar a qualidade de vida do paciente. 


\section{a) Índice de Severidade da Psoríase por Área (PASI)}

No PASI, as placas de psoríase são analisadas segundo três critérios: eritema (E), infiltração (I) e descamação (D) sendo estabelecido a gravidade do critério através de uma escala ordinal de intensidade de 0 (para o não envolvimento) a 4 (para o envolvimento grave).

O corpo é dividido em quatro regiões: cabeça (C), membros superiores (MS), tronco (T), e membros inferiores (MI). Em cada uma dessas áreas $(\mathrm{A})$, a fração da superfície total acometida é graduada ordinalmente de 0 (nenhum envolvimento) a 6 (envolvimento acima de 90\%).

As várias regiões do corpo foram ponderadas para refletir suas respectivas proporções de área de superfície corporal. Sendo assim, a área afetada e as características das lesões foram analisadas através de uma fórmula que resulta em um escore que varia de 0 a 72 pontos.

\section{b) Índice de Qualidade de Vida em Dermatologia (DLQI)}

O DLQI consiste em um questionário autoaplicável, constituído de dez questões, cada qual com quatro respostas possíveis, recebendo um score de 0 a 3 para cada uma delas (nada: 0 , um pouco: 1, muito: 2, muitíssimo: 3). Este questionário avalia sintomas e sentimentos (item 1 e 2), atividades diárias (3 e 4), atividades de lazer, práticas esportivas (5 e 6), trabalho (7), relações interpessoais (8 e 9) e o tratamento (10) o que resulta, ao final do questionário, em um score que pode variar de 0 a 30 . Tal score foi agrupado de acordo com o impacto na qualidade de vida (0-1: nada, 2-5: pouco, 6-10: moderado, 11-20: muito e 21-30: muitíssimo) (TORRES RAT, et al., 2011).

Após a avaliação clínica e exame dermatológico foram solicitados exames laboratoriais tais como hormônio estimulante de tireoide (TSH), T4 livre, Anti-tiroperoxidase (anti-TPO), Antitireoglobulina. Tais exames foram realizados no laboratório de análises clínicas do CCBS UEPA. Para tal análise, foram considerados os valores de referência para o TSH os resultados entre 0,27 e 4,2 mU/L, e para o T4 livre, foram avaliados como dentro da normalidade os valores entre 0,89 e 1,76 ng/dL (SANTORO D, et al., 2017)

\section{Uma vez efetuada a análise laboratorial: Análise estatística}

Todos os resultados foram registrados em banco de dados do Microsoft Excel 2007 para posterior análise estatística com o programa BioStat 5.0. As hipóteses foram avaliadas pelo teste estatístico T de Student de acordo com os valores estatística obtidos, adotando-se um nível de significância em 95\%., adotou-se o nível de significância $\alpha=0,05$ ou $5 \%$, sinalizando com asterisco $\left({ }^{*}\right)$ os valores significantes.

\section{RESULTADOS}

Foram estudados 40 pacientes com psoríase e 20 pacientes controles. Sobre os pacientes com psoríase, verificou-se que 19 eram do sexo feminino e 21 do sexo masculino. Dentro dos pacientes controles 14 eram do sexo feminino e 6 do sexo masculino. A média da idade dos pacientes com psoríase foi de 51,7 anos e 0 desvio padrão de 13 anos. Por outro lado, a média dos pacientes controle foi de 30,1 anos e o desvio padrão de 13 anos. Observou-se diferença significativa $(p<0,0001)$ entre as idades dos pacientes do grupo de psoríase quando comparados ao controle, sendo o primeiro grupo composto por idades mais elevadas.

Acerca do perfil tireoidiano dos pacientes com psoríase, observou-se que 13 (32,5\%) possuíam história familiar de doença tireoidiana e $3(7,5 \%)$ possuíam história pessoal de doença na glândula, enquanto que os controles possuíam $5(25 \%)$ de história familiar e 2 (10\%) de história pessoal positiva.

Os níveis de TSH do grupo com psoríase foram alterados em 11,7\% dos pacientes e tiveram uma média de 2,2 e desvio padrão de 1,2, enquanto que os níveis do grupo controle apresentaram uma média de 2,8 e desvio padrão de 2,7. Dentro do grupo com psoríase o T4 Livre foi alterado em 38,3\% e obtiveram uma média de 1,6 e desvio padrão de 2,1. O grupo controle apresentou média de 5.1 e desvio padrão de 3.7. Portanto, observou-se diferença significante $(p<0,0004)$ entre os grupos (Tabela 3). Acerca dos anticorpos, o grupo de psoríase apresentou média de $7.3 \pm 2.1$ de anti-TPO e $1.7 \pm 21.0$ de Anti-tireoglobulina e foram alterados em respectivamente $23,3 \%$ e $13,3 \%$ dos pacientes. Quando comparado com o grupo controle, observou-se 
diferença significante nos os níveis de anti-TPO entre os grupos, com $p$ valor de 0,0015. Sendo assim, verificou-se que $61,7 \%$ dos pacientes estudados tinham alguma alteração entre os níveis laboratoriais tireoidianos. Portando houve incidência significante de presença de alterações tireoidianas entre os pacientes.

Tabela 3 - Características clínicas e epidemiológicas dos pacientes do estudo.

\begin{tabular}{lccc}
\hline Perfil epidemiológico & Psoríase & Controle & p-valor \\
\hline $\mathbf{N}$. & $\mathbf{4 0}$ & $\mathbf{2 0}$ & \\
Idade (anos) & $51.7 \pm 13.0$ & $30.1 \pm 13.0$ & $<\mathbf{0 . 0 0 0 1}$ \\
Sexo (F/M) & $19 / 21$ & $14 / 06$ & 0.1688 \\
História familiar & $13(32.5 \%)$ & $5(25.0 \%)$ & 0.5501 \\
História de doença na tireóide & $3(7,5 \%)$ & $2(10.0 \%)$ & 0.8678 \\
TSH & $2.2 \pm 1.2$ & $2.8 \pm 1.7$ & 0.0653 \\
T4 Livre & $1.6 \pm 2.1$ & $5.1 \pm 3.7$ & $\mathbf{0 . 0 0 0 4}$ \\
anti TPO & $7.3 \pm 2.1$ & $1.7 \pm 2.3$ & $\mathbf{0 . 0 0 1 5 ^ { \star }}$ \\
Anti-tireoglobulina & $1.7 \pm 21.0$ & $2.9 \pm 3.7$ & 0.0764 \\
Vitamina D & $25.7 \pm 10.2$ & $27.9 \pm 7.9$ & 0.1999 \\
\hline
\end{tabular}

Legenda: F: Feminino, M: Masculino, TSH: Hormônio estimulante da tireóide, TPO:Anti-tiroperoxidase Fonte: Oliveira BL, et al., 2019.

Com relação ao PASI dos pacientes com psoríase, foi observada uma média de 9,5 e desvio padrão de 7,8 naqueles com alterações tireoidianas e uma média de 9,8 e desvio padrão de 10,3 naqueles sem alterações tireoidianas (p-valor: 0,454). Por outro lado, o DLQI apresentou média de 6 e desvio padrão de 7,6 nos pacientes com alterações tireoidianas e média de 7,5 e desvio padrão de 7,7 naqueles sem alterações tireoidianas ( $p$-valor: 0,268 ). Portanto, não houve diferença significante entre os valores dos scores de PASI e DLQI nos pacientes com psoríase em relação as alterações tireoidianas. Além disso, quando utilizado a correlação de Pearson, também não se obteve correlação significante entre os níveis hormonais tireoidianos e os scores PASI e DLQI.

Gráfico 1 - Relação entre o PASI e as alterações tireoideanas. Teste T-student. PASI PASI x Alterações tireoideanas

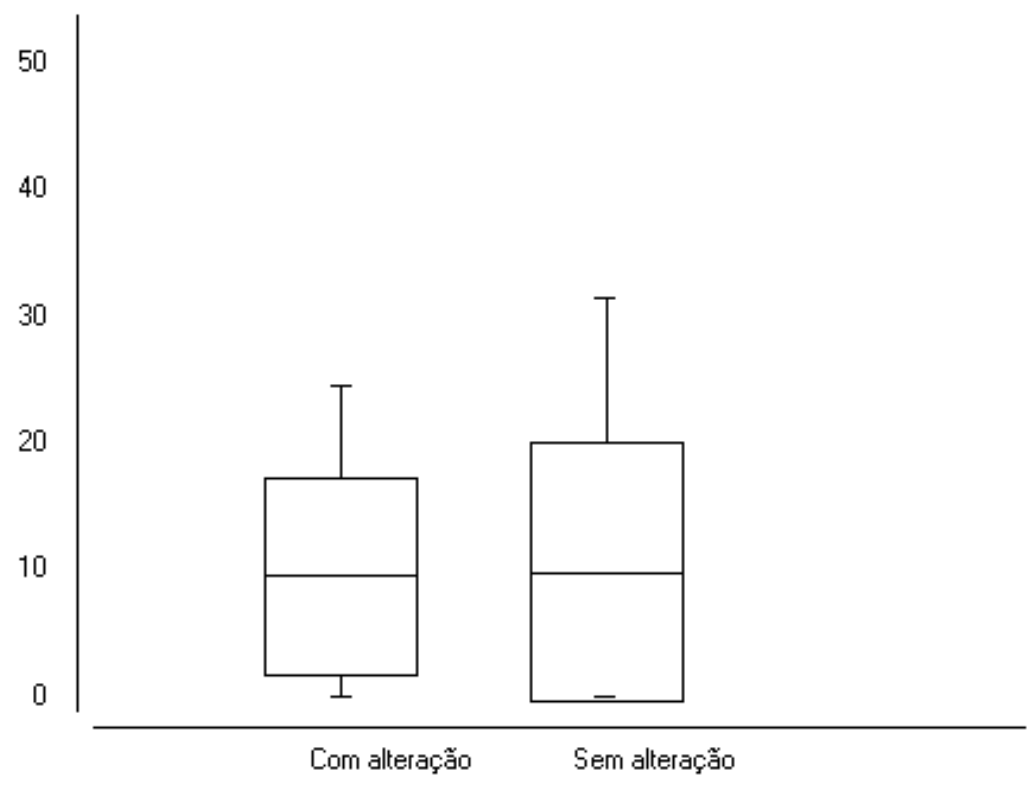

Fonte: Oliveira BL, et al., 2019. 
Tabela 4 - Correlação entre hormônios tireoideanos e as pontuações no PASI e no DLQI

\begin{tabular}{lcccc}
\hline Correlação de Pearson & TSH & T4 Livre & anti TPO & $\begin{array}{c}\text { Anti- } \\
\text { tireoglobulina }\end{array}$ \\
\hline PASI & & & & \\
Coeficiente de correlação & $-0,0150$ & $-0,1307$ & $-0,0946$ & $-0,1264$ \\
p-valor & 0,9270 & 0,4213 & 0,5616 & 0,4370 \\
$\mathrm{n}$ & 40 & 40 & 40 & 40 \\
\hline DLQI & & & & \\
Coeficiente de correlação & $-0,0988$ & $-0,1607$ & $-0,0454$ & $-0,1525$ \\
p-valor & 0,5723 & 0,3565 & 0,7955 & 0,3819 \\
$\mathrm{n}$ & 35 & 35 & 35 & 35 \\
\hline
\end{tabular}

Legenda: TSH: Hormônio estimulante da tireóide, T4: tetraiodotironina, PASI: Índice de Severidade da Psoríase por Área, n: Número de Pacientes, DLQI: Índice de Qualidade de Vida em Dermatologia Fonte: Oliveira BL, et al., 2019.

\section{DISCUSSÃO}

O presente estudo observou um aumento significativo dos níveis de anti-TPO entre os pacientes do grupo com psoríase quando comparados com o grupo controle. Realizou-se uma meta análise a qual também revelou uma associação entre a positividade do anti-TPO e prevalência de psoríase (KHAN SR, et al., 2017). Além disso, este estudo também identificou que hipertireoidismo e hipotireoidismo também podem estar associados à psoríase.

Sugere-se que o aumento nos níveis de hormônios tireoidianos, bem como o aumento dos níveis de antiTPO podem ativar o aumento da produção de fator de crescimento epidérmico, o qual é um fator importante para a proliferação de queratinócitos. Consequentemente, se portando como mecanismo relevante no desenvolvimento das manifestações clínicas da psoríase. (ZOABI A, et al., 2012)

Takir observou que, dentre os pacientes com doenças tiroidianas, aqueles que apresentavam psoríase possuíam principalmente alterações autoimunes em suas glândulas. Sendo assim, corroborando a relação entre a psoríase e a doença autoimune tireoidiana (TAKIR M, et al). Ademais, Kiguzarde descreveu a associação significativa entre pacientes com psoríase e tireoidite de Hashimoto, a qual se trata da doença autoimune tireoidiana mais prevalente no mundo (KIGURADZE T, et al., 2017).

Por outro lado, em estudos que observaram esta relação entre psoríase e alterações tireoidianas, também foi relatada uma maior associação quando a psoríase também tinha acometimento articular. Os autores relacionam esta associação ao maior estado inflamatório o qual o paciente com artrite psoriática se encontra, o qual apresenta níveis mais elevados de proteína c-reativa e interleucinas, como a Interleucina-6, Interleucina-2 e Interleucina-1 (WANG SH, et al., 2018). Além disso, a presença de alterações tireoidianas leva a uma doença com mais articulações acometidas e com uma maior duração, gravidade e menor resposta aos tratamentos convencionais. Portanto, autores afirmam que a investigação de alterações tireoidianas deve ser realizada durante o acompanhamento do paciente com psoríase, principalmente naqueles com artrite psoriática. Sendo assim, devendo ser realizada a dosagem de hormônios e anticorpos tireoidianos periodicamente para rastreio de possíveis doenças correlacionadas. (WANG SH, et al., 2018; ANTONELLI A, et al., 2006).

Tendo em vista a relação descrita entre fatores imunes e as duas doenças, Antonelli A, et al. (2008) estudou a associação entre os níveis séricos das quimiocinas CXCL10 e CCL2 entre os pacientes com artrite psoriática com doença autoimune na tireoide e pacientes sem autoimunidade tireoidiana. Observou-se níveis mais elevados destas quimiocinas nos pacientes que possuíam artrite psoriática com acometimento autoimune da glândula. Todavia o autor relata que mais estudos devem ser realizados para definir o potencial 
de determinação de prognóstico destas quimiocinas em relação aos pacientes com artrite psoriática (ANTONELLI A, et al, 2008). Rufilli afirma que devido ao perfil de tais quimiocinas, a acentuação da resposta imune Th1 deve ser o mecanismo de relação entre a psoríase e as doenças autoimune tireoidianas (RUFFILLI I, et al, 2017).

Os níveis de T4 livre no presente estudo, observados entre os pacientes com psoríase, foram significativamente menores quando comparados com os pacientes controles. Por outro lado, Gul U, et al. (2009) relatou o aumento dos níveis de T4 livre em paciente com psoríase vulgar quando comparados com pacientes sem a doença (GUL U, et al, 2009). Ademais, Arican relatou que os pacientes que tinham maiores níveis de T3 livre e T4 livre eram os que possuíam PASI mais elevados (ARICAN O, et al, 2004).

Contrariamente, em estudo realizado por Robati RM, et al. (2013), foi analisado o PASI, o T3 livre e T4 livre dos pacientes com psoríase antes e depois do tratamento e verificou-se que, mesmo após o tratamento e diminuição do PASI, os níveis de hormônios tireoidianos não alteravam significativamente. Portanto, não apresentando correlação entre os níveis hormonais tireoidianos e a gravidade da psoríase. (ROBATI RM, et al., 2013).

Dessa forma, concordando com o presente estudo, visto que igualmente não foi observado diferença ou correlação significante entre os valores do score PASI ou DLQI em relação as alterações tireoidianas. Por outro lado, foi observado que após o tratamento com Propiltiouracil em pacientes com psoríase em placa, estes apresentavam melhora dos sintomas e diminuição do score PASI (MALLIGARJUNAN H, et al., 2011).

Estudos revelaram um menor nível de T3 livre nos pacientes com psoríase quando comparados com os pacientes sem a doença (HANSEN PR et al., 2019). Todavia, diversos outros trabalhos não encontraram diferenças significativas entre os níveis de T3 livre entre os pacientes com psoríase e os pacientes do grupo controle (LAI YC e YEW YW, 2016).

Observou-se que o hormônio T3 possui receptores na pele, os quais participam da síntese dos queratinócitos. Além disso, tais hormônios também participam da regulação dos fatores de crescimento epidérmico. Portanto, drogas antitireoidianas como o Propiltiuracil podem ter benefício no controle de produção e diferenciamento celular na pele (ARICAN O, et al., 2004).

Sobre o hormônio TSH, não se observou diferença significativa entre os valores do grupo com psoríase em comparação aos do grupo controle. Portando concordando com Axita, a qual também não observou relação significativa do valor de TSH entre os grupos, além de também rejeitar a associação dos valores de T3 e T4 com a psoríase (VANI AC, et al., 2017).

O presente estudo não observou relação significativa entre positividade de Antitireoglobulina entre os pacientes com psoríase. Vassilatou E, et al. (2017) igualmente relatou não haver maior positividade de tal anticorpo em pacientes com psoríase. Além disto, o mesmo autor também negou a associação da positividade de outros marcadores de doença autoimune tireoidianas em relação à psoríase. (VASSILATOU E, et al., 2017).

O presente estudo apresentou diferença significativa entre as idades dos pacientes com psoríase em comparação com os pacientes controles. O primeiro grupo apresentou uma média de idade significativamente maior em comparação com os pacientes sem a doença.

\section{CONCLUSÃO}

Tendo em vista os resultados apresentados neste estudo, conclui-se que existe uma relação significante entre maiores níveis de anti-tiroperoxidase nos pacientes com psoríase quando comparados com o grupo controle. Além disto, também foi observado aumento significante dos níveis de T4 livre entre os pacientes deste grupo. Não foram encontradas relações significantes entre o Índice de Severidade da Psoríase por Área (PASI) e alterações tireoidianas. Todavia, maiores estudos devem ser realizados para estabelecer concretamente a associação entre a psoríase e suas formas com as doenças autoimunes da tireoide.

REAS/EJCH | Vol.12(2) | e2378 | DOI: https://doi.org/10.25248/reas.e2378.2020 Página 7 de 8 


\section{REFERÊNCIAS}

1. ANTONELLI A, et al. High prevalence of thyroid autoimmunity and hypothyroidism in patients with psoriatic arthritis. J Rheumatol. 2006 Oct;33(10):2026-8. [PMID: 177014017].

2. ANTONELLI A, et al. High values of alpha (CXCL10) and beta (CCL2) circulating chemokines in patients with psoriatic arthritis, in presence or absence of autoimmune thyroiditis Autoimmunity. $2008 \mathrm{Nov}$;41(7):537-42. [PMID:18855195].

3. ARICAN O, et al. The effect of thyroid hormones in psoriasis vulgaris. Indian $\mathrm{J}$ Dermatol Venereol Leprol., 2004;70(6):353-6. [PMID:27050092].

4. CATHER JC, et al. Psoriasis and Psoriatic Arthritis. J Clin Aesthet Dermatol. 2017 Mar;10(3): S16-S25. Epub 2017 Mar 1. [PMID:28360971].

5. FALLAHI P, et al. Increased incidence of autoimmune thyroid disorders in patients with psoriatic arthritis: a longitudinal follow-up study. Immunol Res., 2017 Jun;65(3):681-686. [PMID: 28101810].

6. GNANARAJ P, et al. Downregulation of involucrin in psoriatic lesions following therapy with propylthiouracil, an antithyroid thioureylene: immunohistochemistry and gene expression analysis. Int J Dermatol. 2015;54(3):302-6. [PMID:25267471].

7. GUL U, et al. Autoimmune thyroid disorders in patients with psoriasis. Eur J Dermatol. 2009;19(3):221-3. [PMID:19251565].

8. HANSEN PR, et al. Thyroid function in psoriasis. Br J Dermatol. 2019 Jan 10. [PMID:30629295].

9. HERNÁNDEZ-VÁSQUEZ A, et al. Psoriasis in Latin America and the Caribbean: a systematic review. J Eur Acad Dermatol Venereol. 2017;31(12):1991-1998. [PMID:28608530].

10. KHAN SR, et al. The association of autoimmune thyroid disease (AITD) with psoriatic disease: a prospective cohort study, systematic review and meta-analysis. Eur J Endocrinol. 2017 Oct;177(4):347-359. [PMID:28747386].

11. KIGURADZE T, et al. Evidence for the association of Hashimoto's thyroiditis with psoriasis: a cross-sectional retrospective study. Int J Dermatol. 2017;56(5):553-556. [PMID: 28217937].

12. KUCHEL J, et al. Short-term resolution of psoriasis after total thyroidectomy for euthyroid multinodular goitre. Australas J of Dermatol 2002 Aug;43(3):214-7. [PMID:12121402].

13. LAI YC, YEW YW. Psoriasis and thyroid profile: Analysis of the U.S. National Health and Nutrition Examination Survey database. Indian J Dermatol Venereol Leprol, 2016 May-Jun;82(3):310-2. [PMID: 27088936].

14. MALLIGARJUNAN H, et al. Impact of propylthiouracil on quality of life in psoriasis patients. Indian J Med Sci. 2011 Aug;65(8):331-6. [PMID:23442529].

15. ROBATI RM, et al. Association of psoriasis severity with serum prolactin, thyroid hormones, and cortisol before and after treatment. ScientificWorldJournal. 2013; 282013:921819. [PMID:24288511].

16. ROMAN II, et al. The role of hormones in the pathogenesis of psoriasis vulgaris. Clujul Med. 2016;89(1):11-8. [PMID:27004020].

17. RUFFILLI I, et al. Psoriasis, Psoriatic Arthritis, and Thyroid Autoimmunity Front Endocrinol (Lausanne). 2017 Jun 19 ; 8:139. [PMID:28674524].

18. SANTORO D, et al. Autoimmune Thyroiditis and Glomerulopathies. Front Endocrinol (Lausanne).2017;8:119. [PMID: 28626447].

19. SCHÖN MP, BOEHNCKE H. Psoriasis. N Engl J Med 2005 May 5;352(18):1899-912. [PMID:15872205].

20. TAKIR M, et al. Skin findings in autoimmune and nonautoimmune thyroid disease with respect to thyroid functional status and healthy controls. Turk J Med Sci. 2017 Jun 12;47(3):764-770. [PMID:28618767].

21. TORRES RAT, et al. Comparação entre questionários de qualidade de vida e sua correlação com a evolução clínica de pacientes com psoríase. An Bras Dermatol 2011; 86(1): 45-9. Doi: 10.1590/S0365-05962011000100005.

22. VANI AC, et al. A Study of Thyroid Profile in Patients with Psoriasis. National Journal of Laboratory Medicine. 2017, Jul, Vol-6(3). DOI:10.7860/NJLM/2017/29047:2233

23. VASSILATOU E, et al. No association of psoriasis with autoimmune thyroiditis. J Eur Acad Dermatol Venereol, 2017 Jan;31(1):102-106. [PMID: 27324349].

24. WANG SH, et al. Increased risk of incident thyroid diseases in people with psoriatic disease: A cohort study. J Am Acad Dermatol. 2018 Dec 5. pii: S0190-9622(18)32985-2. [PMID: 3052850].

25. WU JJ, et al. The association of psoriasis with autoimmune diseases. J Am Acad Dermatol. 2012;67(5):924-30. [PMID:22664308].

26. ZOABI A, et al. Prevalence of thyroid abnormalities among psoriatic patients. Harefuah. 2012 Oct;151(10):566-9, 605-6. [PMID: 23316662]. 